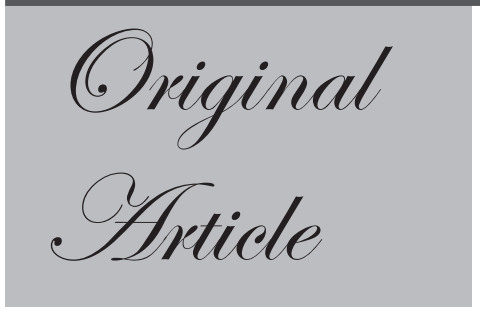

\title{
Hepatitis B Vaccination Amongst Medical Students: Is There a Need to Review Seroconversion Post Vaccination?
}

\author{
Premashis Kar, Prabhat Gautam Roy, Ayush Gupta, \\ Rajib K Hazam, Vijay Karra
}

Department of Medicine,

ABSTRACT

Maulana Azad Medical College,

University of Delhi,

New Delhi, India

Corresponding Author: Dr Premashis Kar

Email:premashishkar@gmail.com

Background: Seroconversion rates following Hepatitis B vaccination all around the world have been reported to be $>90 \%$ in general population. However, a few studies have shown that seroconversion is less amongst Health Care Workers which can be attributed to various reasons. Medical Students are at a high risk of acquiring HBV during their clinical experience and the rate of seroconversion following vaccination was not reported previously in Indian Medical Students. Therefore this study was conducted to detect AntiHbS titres and to look into factors associated with failure of vaccination in medical students of Maulana Azad Medical College in New Delhi.

Methods: An Institutional study was conducted on 89 Medical Students with prior Hepatitis B vaccination. Their AntiHbS titres were calculated and associations of non-protective levels were sought with various factors. Results: $74.2 \%$ (66/89) students developed protective levels of AntiHbS titres $(=10 \mathrm{mIU} / \mathrm{mL})$ whereas $25.8 \%(23 / 89)$ had AntiHbS $<10 \mathrm{mIU} /$ $\mathrm{mL}$. Significant associations of non-protective AntiHbS titres $(<10 \mathrm{mIU} /$ $\mathrm{mL}$ ) were found with BMI $=23.5$ (p-value 0.014), Alcohol Consumption ( $p$-value 0.032) and Waist Hip Ratio $=0.85$ in females ( $p$-value 0.007). Associations with Waist Hip Ratio $=0.95$ in males ( $p$-value 0.056 ), Tobacco Consumption ( $p$-value 0.969), past history of jaundice (p-value 0.474), Occupational exposure ( $\mathrm{p}$-value 0.553 ) and history of blood transfusion (p-value 0.298 ) and Gender were statistically non-significant.

Conclusions: It was concluded that the rate of seroconversion in medical students was less than that reported for general population. Therefore, it is crucial to check AntiHbS antibody titres post vaccination and to give a booster dose of Hepatitis B vaccination to those with non-protective titres.

KEYWORDS: Anti HbS seroconversion, factors affecting seroconversion, Hepatitis B vaccination, Indian Medical Students. 


\section{Introduction}

Hepatitis B virus (HBV) causes liver disease and up to 2 billion people worldwide have been infected with the virus at some point in their lives. ${ }^{1}$ It can cause both acute and chronic liver disease. If primary infection occurs, about $95 \%$ of immunocompetent adults experience a self-limiting disease while the remaining $5 \%$ end up becoming chronic carriers of HBV. There are about 400 million carriers of Hepatitis B worldwide. India accounts for $10-15 \%$ of entire pool of Hepatitis B carriers in the world. ${ }^{2}$ There are varying reports of $\mathrm{HbSAg}$ positivity in India ranging from 2-4.7\%. ${ }^{3,4}$ People who develop chronic HBV infection are at an increased risk for hepato-cellular carcinoma, cirrhosis and death. They also serve as a means for transmitting infection to other unvaccinated or under-vaccinated individuals. Following World Health Organization (WHO) recommendations India has implemented the vaccine in it's children's immunization programme. For adults, Hepatitis B vaccine is recommended in certain high-risk groups like medical students and healthcare workers. Following vaccination, it has been seen in general population globally that 90 95\% individuals develop protective levels of antibodies to $\mathrm{HbSAg}$ (defined as AntiHbS10 $\geq \mathrm{mIU} / \mathrm{mL}$ ) after three doses. ${ }^{5}$ The remaining are cases of failure of immunization. However, there is hardly any study available from the Indian scenario, which mentions about seroconversion rate following Hepatitis B vaccination amongst Medical students. A data in this regard is highly desirable as an average Indian medical student who is exposed to a large number of patients has inappropriate means and measures to follow the WHO standard universal precautions before attending to each patient. Thus, he becomes very susceptible to an occupational exposure to Hepatitis B virus and its consequences. He also becomes a source of spreading HBV infection to the patients. There have been a few studies which indicate that risk factors for failure of immunization may be increasing age, ${ }^{6,7,8}$ male sex, ${ }^{6,9}$ obesity, ${ }^{10}$ tobacco smoking, ${ }^{9,11,12}$ alcoholism, renal disease, ${ }^{13} \mathrm{HIV}$ infection and other immune compromising conditions. ${ }^{14}$ This study focuses on medical students who have had prior vaccination against Hepatitis B and looks into their seroconversion rate. It also explores the reasons for non-conversion.

\section{Material \& Methods}

This study was an cross sectional type of observational study. It was conducted from May 2014 to July 2014 at Maulana Azad Medical College and Lok Nayak Hospital, New Delhi. Study Population comprised of Students of Maulana Azad Medical College and Lok Nayak Hospital with prior vaccination against Hepatitis B. Sample size for this study was 89 students.

\section{Criteria for inclusion}

Students who had prior vaccination against Hepatitis-B.

\section{Ethical Considerations}

An approval from Institutional Ethics Committee, Maulana Azad Medical College and associated Lok Nayak Hospital \& GB Pant Hospital. The objective of the study was explained to all participants, and their consent was taken. Patients who fulfilled the above criteria were included in the study after taking consent. A detailed demographic, clinical history and other information of the patients were recorded in predesigned and pretested proforma.

\section{Collection of samples}

Blood sample was collected in red top vacuotainers following universal precautions. Serum was centrifuged and stored at $-70^{\circ} \mathrm{C}$.

\section{Quantification of Anti HBs by ELISA}

Samples were tested for Anti $\mathrm{HbS}$ antibody titres using Anti-HBs (Quantitative) ELISA kit-BXEO752A: Fortress diagnostics.

\section{Statistical Analysis}

Students' data were collected through predesigned and pretested proforma filled by them. Analysis was done on data collected regarding Height \& Weight (BMI was calculated), Waist circumference \& Hip circumference 
(Waist Hip Ratio [WHR] was calculated. WHR $\geq 0.95$ for males and $\geq 0.85$ for females was tested for significant association with AntiHbS titres.), Previous exposure to Hepatitis B, Tobacco consumption, Alcohol consumption, Occupational exposure to Hepatitis B \& History of previous Blood transfusion.

Written consent was obtained and they were informed about the purpose, nature of study and various investigations to be carried out for the study. Statistical Analysis was done and Quantitative AntiHbS titres were determined. Chi-square test or Fischer's test as applicable was used to test statistical significance of AntiHbS titres with various parameters analyzed. The data was considered significant when $\mathrm{p}$-value $<0.05$.

\section{Results}

\section{Demographic Profile}

Out of 89 subjects in this study 47 (53\%) were Male and $42(47 \%)$ were Female. The mean age of subjects was $20.18+/-1.16$

\section{Analysis of Quantitative Anti HBs Titres}

Serum analysis of the study participants showed that $66 / 89$ (74.2\%) had developed protective levels ( $\geq 10 \mathrm{mIU} / \mathrm{mL}$ ) of AntiHbS antibody while 23/89 (25.8\%) did not develop protective levels. Gender wise distribution of response to vaccination revealed that $70 \%$ males converted whereas in females the conversion rate was $79 \%$. The Geometric Mean titre was $40.97 \mathrm{mIU} / \mathrm{mL}$.

\section{Factors playing a role in seroconversion}

Various factors were tested for their association with levels of AntiHbS Antibody titres developed after Hepatitis B vaccination. $B M I \geq 23.5$ was found to be significantly associated with non-protective levels of AntiHbS antibody titres. (p-value- 0.014). Waist Hip Ratio (WHR) $\geq 0.95$ in Males did not have any significant effect on levels of AntiHbS antibodies ( $\mathrm{p}$ value $>0.05$ ). However, WHR $\geq 0.85$ in Females had a significant association with non-protective levels of Anti $\mathrm{HbS}$ antibody titres (p-value

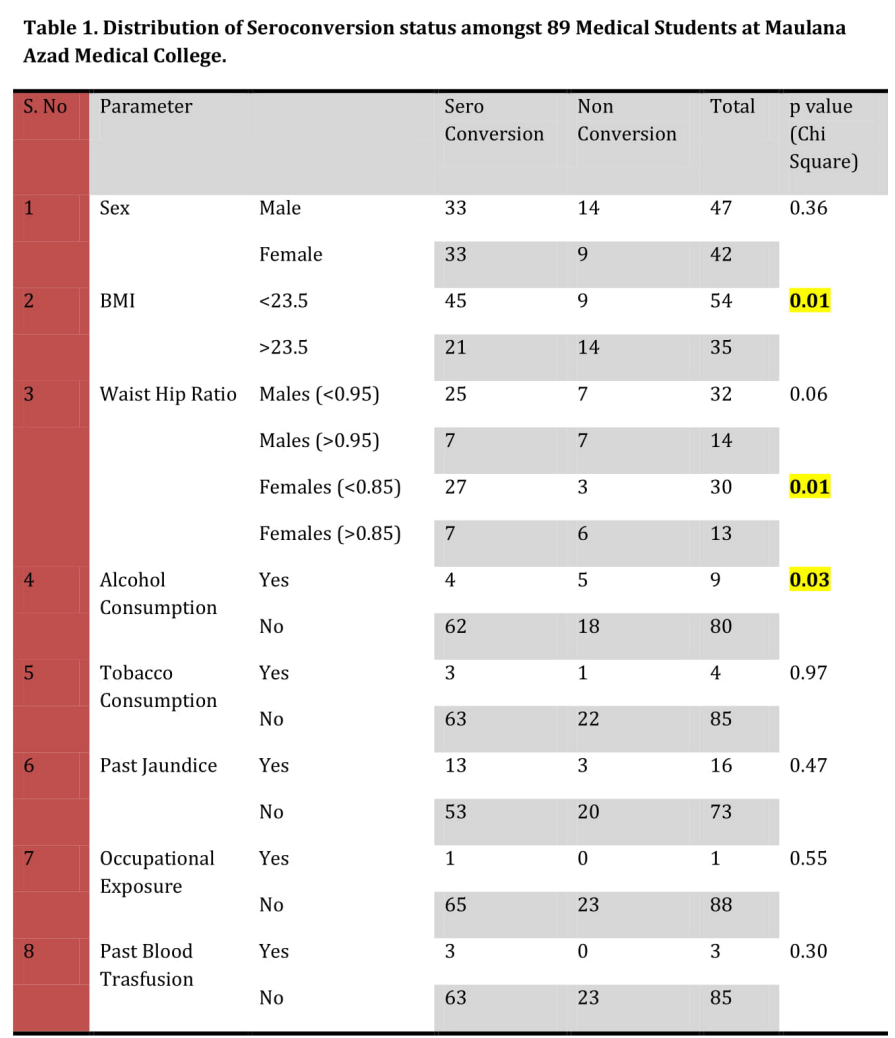

$<0.01)$. Alcohol consumption was significantly related with non-protective Anti $\mathrm{HbS}$ antibody titres (p-value 0.03). The effect of smoking, past history of jaundice, occupational exposure to Hepatitis B and prior blood transfusion was not found to be statistically significant (p-value $0.969,0.474,0.553 \& 0.298$ respectively). A statistical correlation between gender and antibody titres could not be established (p-value 0.306).

\section{Discussion}

This study was designed to detect the response following Hepatitis B vaccination amongst medical students in Indian condition and found that out of 89 vaccinated students who were tested, 23 students $(25.8 \%)$ had non-protective levels (AntiHbS $<10 \mathrm{mIU} / \mathrm{mL}$ ) while 66 students $(74.2 \%)$ had protective levels ( $\geq 10 \mathrm{mIU} / \mathrm{mL})$ of antibody titres. In a similar study in Russia by Tkachenko LI Et.al it was found that immunological response was poor in $20.4 \%$ medical students. ${ }^{15}$ Zeeshan et.al also conducted a similar study in medical students and healthcare workers in Pakistan and found a poor immunological response ranging from $9.1 \%$ in subjects less than 25 years of age to $63 \%$ in subjects 
greater than 50 years of age. ${ }^{16}$ Similar results were also reported by Roome AJ et $\mathrm{al}^{10}$ and SangfeltP et al ${ }^{17}$.

Medical students with non-protective levels $(<10 \mathrm{mIU} / \mathrm{mL})$ were advised repeat vaccination as per CDC guidelines. ${ }^{18}$

The study tried to look into this response and find out the reasons for the sub-normal values of AntiHbS titres following vaccination. In this study it was observed that $30 \%$ males did not develop protective levels whereas among females, only $21 \%$ had non-protective levels. Even though males had a poor immunological response, a statistical association could not be established between gender and AntiHbS levels in this study ( $\mathrm{p}$-value $>0.05$ ) probably because of a limited sample size. Correlation with increasing weight was also tested. BMI $\geq 23.5 \%$ Waist Hip ratio $($ WHR $) \geq 0.95$ in males and $\geq 0.85$ in females were taken as indicators of increased weight. It was found that BMI $\geq 23.5$ was significantly associated with non-protective levels of antibodies (p-value 0.014). Waist hip ratio $\geq 0.95$ was not found to be significantly associated with poor response in Males ( $p$-value $>0.05$ ). However, it was observed that a significant number of females with WHR $\geq 0.85$ had non-protective levels of AntiHbS (p-value 0.007). Further, an association of immunological response with alcohol consumption was also explored. It was found that alcohol consumption was significantly related with poor antibody response $<10 \mathrm{mIU} /$ $\mathrm{mL}$ ( $\mathrm{p}$ value 0.03 ). It has been established previously that cirrhosis is a risk factor for diminished immunological response. ${ }^{19,20}$ Alcohol has been incriminated as a risk factor due to its ability to cause cirrhosis. In this study, no student had cirrhosis and therefore we could conclude that alcohol consumption can independently be a risk factor for non-conversion following HBV vaccination. Smoking was also tested for association with poor immunological response and it was not found to be a factor leading to diminished antibody response ( $\mathrm{p}$-value $>0.05$ ). This can probably be attributed to the fact that our study included individuals aged 19-24 years and none of them had an exposure to tobacco smoking long enough to affect their immunological response. The study also looked into the association of poor immunological response with Past history of Jaundice, Blood transfusion and Occupational exposure to Hepatitis B infection. All of the above were found to be non significant. (p-values 0.474, 0.298 and 0.553 respectively).

In various studies around the world, it has been found that the factors probably associated with subnormal response following vaccination are Male sex, ${ }^{6,9}$ increasing age, ${ }^{6,78}$ smoking, ${ }^{6,11,12}$ obesity, ${ }^{10}$ impaired immune reactivity, ${ }^{14}$ patients on dialysis ${ }^{13}$ and genetic factors like HLA Haplotype [DQB1*0604; DQA1*0102; DRB $1 * 1302] .{ }^{21}$

However, the authors acknowledge that this study was conducted in a relatively smaller sample size, which can affect statistical associations. Also, this study included medical students whose ages varied from 19-24 years thereby limiting the prospect of taking into account possible effects of age on the various factors studied.

\section{Conclusions}

From the findings of this study, it can be concluded that rate of seroconversion in medical students after vaccination against Hepatitis B in this study was $74.2 \%$. It was found to be lower than that reported in similar studies elsewhere in the world (Pakistan- 86\% \& Russia- 79.6\%). Therefore, it is crucial to check AntiHbS antibody titres post vaccination and to give a booster dose of Hepatitis B vaccination to those with non-protective titres (AntiHbS levels $<10 \mathrm{mIU} / \mathrm{mL}$ ). This strategy will ensure safety at work by reducing nosocomial transmission. The factors found responsible for non-conversion (AntiHbS titres $<10 \mathrm{mIU} / \mathrm{mL}$ ) in both the genders were BMI $\geq 23.5$ (p-value 0.014) and alcohol consumption (p-value 0.032). Waist Hip ratio $\geq 0.85$ was found to be an added factor in females leading to non-conversion (p-value 0.007). However, since the sample size of this study was small, a more extensive analysis in a large-scale study is needed to further test the associations found in this study.

\section{References}

1. M.L. Schilsky, Hepatitis B “360", Department of Medicine and Surgery, Section of Digestive Diseases and Section of Transplantation and Immunology, Yale University School of Medicine, New Haven, Connecticut

2. Lahariya et al, Indian J Public Health, 2013; and Lahariya C, Indian J medical Research, 2014 
3. Abraham P. Viral Hepatitis in India.Clin Lab Med. 2012;32(2):159-74.

4. Thyagarajan SP, Jayaram S, Mohanavalli BIn: Sarin SK, Singal AK, (Eds)Prevalence of HBV in general population in India,Hepatitis B in India: problems and prevention. New Delhi: CBS;1996.pp.5-16.

5. Batham A, Narula D, Toteja T, Sreenivas V, Puliyel JM. Sytematic review and meta-analysis of prevalence of hepatitis B in India. Indian Pediatr 2007; 44: 663-74

6. Struve J, Aronsson B, Frenning B, Granath F, von Sydow M, Weiland O. Intramuscular versus intradermal administration of a recombinant hepatitis $\mathrm{B}$ vaccine: a comparison of response rates and analysis of factors influencing the antibody response. Scand J Infect Dis 1992;24(4):423-9.

7. Havlichek D, Jr., Rosenman K, Simms M, Guss P. Agerelated hepatitis $\mathrm{B}$ seroconversion rates in health care workers. Am J Infect Control 1997;25(5):418-20.

8. Clements ML, Miskovsky E, Davidson M, et al. Effect of age on the immunogenicity of yeast recombinant hepatitis $B$ vaccines containing surface antigen $(\mathrm{S})$ or PreS2 $+\mathrm{S}$ antigens. J Infect Dis 1994;170(3):510-6.

9. Senden TF. Response to intradermal hepatitis B vaccination: differences between males and females? Vaccine 1990;8(6):612-3.

10. Roome AJ, Walsh SJ, Cartter ML, Hadler JL. Hepatitis B vaccine responsiveness in Connecticut public safety personnel. Jama 1993;270(24):2931-4.

11. Winter AP, Follett EA, McIntyre J, Stewart J, Symington IS. Influence of smoking on immunological responses to hepatitis B vaccine. Vaccine 1994;12(9):771-2.

12. Wang LY, Lin HH. Ethnicity, substance use, and response to booster hepatitis B vaccination in anti-HBs-seronegative adolescents who had received primary infantile vaccination. J Hepatol 2007;46(6):1018-25.

13. Buti M, Viladomiu L, Jardi $R$, et al. Long-term immunogenicity and efficacy of hepatitis B vaccine in hemodialysis patients. Am J Nephrol1992;12(3):144-7.

14. Tedaldi EM, Baker RK, Moorman AC, et al. Hepatitis $\mathrm{A}$ and $\mathrm{B}$ vaccination practices for ambulatory patients infected with HIV. Clin Infect Dis 2004;38(10):1478-84.

15. Tkachenko LI, Maleev VV, Putrenok LS. Evaluation of seroconversion after vaccination of medical staff against HBV infection. ZhMikrobiolEpidemiolImmunobiol. 2013 Sep-Oct;(5):69-74.e

16. Mohammad Zeeshan1, Kauser Jabeen1, Anita Nausheen Akbar Ali2, Ailia Wilayat Ali2,Saadia Z Farooqui2, Vikram Mehraj1 and Afia Zafar. Evaluation of immune response to Hepatitis B vaccine in health care workers at a tertiary care hospital in Pakistan: an observational prospective study. BMC Infectious Diseases 2007, 7:120 doi:10.1186/14712334-7-120

17. Sangfelt P, Uhnoo I, Reichard O, Weiland O. A low-dose intradermal hepatitis $\mathrm{B}$ vaccine programme in health-care workers and students is highly effective and cost saving: a retrospective follow-up survey in the clinical setting. World J Gastroenterol. Aug 14, 2014; 20(30): 10383-10394.

18. Guidance for Evaluating Health- Care Personnel for Hepatitis B Virus Protection and for Administering Postexposure Management, MMWR 2013; 62(RR10): 1-19; www.cdc.gov/mmwr/pdf/rr/ rr6210.pdf.

19. De Maria N, Idilman R, Colantoni A, Van Thiel DH. Increased effective immunogenicity to high-dose and short-interval hepatitis B virus vaccination in individuals with chronic hepatitis without cirrhosis. J Viral Hepat2001;8(5):372-6.

20. Dominguez M, Barcena R, Garcia M, Lopez-Sanroman A, Nuno J. Vaccination against hepatitis B virus in cirrhotic patients on liver transplant waiting list. Liver Transpl 2000;6(4):440-2.

21. Kristina Cardell Studies on Hepatitis B Vaccination and Factors Associated with the Vaccine Response LiUCtryck, Linköping, Sweden, 2009. 